\title{
Immunohistochemical Mapping of Sensory Nerve Endings in the Human Triangular Fibrocartilage Complex
}

\author{
Susanne Rein MD, PhD, Manuel Semisch, Marc Garcia-Elias MD, PhD, Alex Lluch MD, \\ Hans Zwipp MD, PhD, Elisabet Hagert MD, PhD
}

Received: 17 December 2014/ Accepted: 12 May 2015/Published online: 30 May 2015

(C) The Association of Bone and Joint Surgeons (B) 2015

\begin{abstract}
Background The triangular fibrocartilage complex is the main stabilizer of the distal radioulnar joint. While static joint stability is constituted by osseous and ligamentous integrity, the dynamic aspects of joint stability chiefly concern proprioceptive control of the compressive and directional muscular forces acting on the joint. Therefore, an investigation of the pattern and types of sensory nerve endings gives more insight in dynamic distal radioulnar joint stability.
\end{abstract}

Funding for this study was received from Willi \& Partner AG, Wetzikon, Switzerland (SR), and by the Swedish National Center for Research in Sports (EH).

All ICMJE Conflict of Interest Forms for authors and Clinical Orthopaedics and Related Research ${ }^{\circledR}$ editors and board members are on file with the publication and can be viewed on request.

Clinical Orthopaedics and Related Research ${ }^{\circledR}$ neither advocates nor endorses the use of any treatment, drug, or device. Readers are encouraged to always seek additional information, including FDAapproval status, of any drug or device prior to clinical use.

Each author certifies that his or her institution approved the human protocol for this investigation, that all investigations were conducted in conformity with ethical principles of research, and that informed consent for participation in the study was obtained.

This work was performed at the Department of Anatomy, University of Barcelona, and at the Center for Orthopedics and Trauma Surgery, University Hospital "Carl Gustav Carus," Dresden, Germany.

\section{S. Rein}

Department of Hand and Plastic Surgery, Trauma Center

Bergmannstrost, Halle/Saale, Germany

S. Rein ( $\square)$, M. Semisch, H. Zwipp

Center for Orthopedics and Trauma Surgery, University Hospital

"Carl Gustav Carus", Fetscherstraße 74, 01307 Dresden,

Germany

e-mail: susanne.rein@web.de
Questions/purposes We aimed to (1) analyze the general distribution of sensory nerve endings and blood vessels; (2) examine interstructural distribution of sensory nerve endings and blood vessels; (3) compare the number and types of mechanoreceptors in each part; and (4) analyze intrastructural distribution of nerve endings at different tissue depth.

Methods The subsheath of the extensor carpi ulnaris tendon sheath, the ulnocarpal meniscoid, the articular disc, the dorsal and volar radioulnar ligaments, and the ulnolunate and ulnotriquetral ligaments were dissected from 11 human cadaver wrists. Sensory nerve endings were counted in five levels per specimen as total cell amount/ $\mathrm{cm}^{2}$ after staining with low-affinity neurotrophin receptor p75, protein gene product 9.5 , and S-100 protein and thereafter classified according to Freeman and Wyke.

Results All types of sensory corpuscles were found in the various structures of the triangular fibrocartilage complex with the exception of the ulnolunate ligament, which contained only Golgi-like endings, free nerve endings, and unclassifiable corpuscles. The articular disc had only free nerve endings. Furthermore, free nerve endings were the predominant sensory nerve ending (median, $72.6 / \mathrm{cm}^{2}$; range, $0-469.4 / \mathrm{cm}^{2}$ ) and more prevalent than all other types of mechanoreceptors: Ruffini (median, 0; range, 0 $5.6 / \mathrm{cm}^{2} ;$ difference of medians, 72.6; $\left.\mathrm{p}<0.001\right)$, Pacini

M. Garcia-Elias, A. Lluch

Institut Kaplan, Hand and Upper Extremity Surgery, Barcelona, Spain

\section{E. Hagert}

Department of Clinical Science and Education, Hand and Foot Surgery Center, Karolinska Institutet, Stockholm, Sweden 
(median, 0; range, $0-3.8 / \mathrm{cm}^{2}$; difference of medians, 72.6; $\mathrm{p}<0.001$ ), Golgi-like (median, 0; range, $0-2.1 / \mathrm{cm}^{2}$; difference of medians, 72.6; $\mathrm{p}<0.001$ ), and unclassifiable corpuscles (median, 0 ; range, $0-2.5 / \mathrm{cm}^{2}$; difference of medians, 72.6; $\mathrm{p}<0.001)$. The articular disc contained fewer free nerve endings (median, 1.8 ; range, $0-17.8 / \mathrm{cm}^{2}$ ) and fewer blood vessels (median, 29.8; range, 0-112.2/ $\mathrm{cm}^{2}$; difference of medians: 255.9) than all other structures of the triangular fibrocartilage complex $(\mathrm{p} \leq 0.001$, respectively) except the ulnolunate ligament. More blood vessels were seen in the volar radioulnar ligament (median, 363.62; range, $117.8-871.8 / \mathrm{cm}^{2}$ ) compared with the ulnolunate ligament (median, 107.7; range, $15.9-410.3 / \mathrm{cm}^{2}$; difference of medians: $255.91 ; \mathrm{p}=0.002$ ) and the dorsal radioulnar ligament (median, 116.2; range, 53.9-185.1/ $\mathrm{cm}^{2}$; difference of medians: $247.47 ; \mathrm{p}=0.001$ ). Free nerve endings were obtained in each structure more often than all other types of sensory nerve endings $(p<0.001$, respectively). The intrastructural analysis revealed no differences in mechanoreceptor distribution in all investigated specimens with the numbers available, showing a homogenous distribution of proprioceptive qualities in all seven parts of the triangular fibrocartilage complex.

Conclusions Nociception has a primary proprioceptive role in the neuromuscular stability of the distal radioulnar joint. The articular disc and ulnolunate ligament rarely are innervated, which implies mainly mechanical functions, whereas all other structures have pronounced proprioceptive qualities, prerequisite for dynamic joint stability.

Clinical Relevance Lesions of the volar and dorsal radioulnar ligaments have immense consequences not only for mechanical but also for dynamic stability of the distal radioulnar joint, and surgical reconstruction in instances of radioulnar ligament injury is important.

\section{Introduction}

The distal radioulnar joint is one of the most important and unique articulations in the wrist. The distal radioulnar joint, together with the proximal radioulnar joint, is responsible for the pronosupination movement of the radius and the ulna [14]. Joint stability relies on fine interactions of mechanical and dynamic components. Although static joint stability is constituted by osseous and ligamentous integrity, the dynamic elements of joint stability concern proprioceptive control of the compressive and directional muscular forces acting on the joint $[3,21]$. The core of the distal radioulnar joint stability is best described with the concept of "tensegrity," which entails stability through a synergy of ligament tensile and joint compressive forces [11]. This enables a harmonic rotation of the forearm. The triangular fibrocartilage complex is of primary importance in this synergy $[19,20]$.

Ulnar-sided wrist pain is one of the most common clinical disorders of the wrist and may be dependent on injuries or degenerative disease. At the core of ulnar-sided wrist pain is an afferent painful stimuli from one of the seven structures constituting the triangular fibrocartilage complex, namely the subsheath of the extensor carpi ulnaris tendon sheath, the ulnocarpal meniscoid, the articular disc, and the dorsal and volar radioulnar, ulnolunate, and ulnotriquetral ligaments [10].

The general innervation of the triangular fibrocartilage complex has been described and consists of innervation by dorsal sensory branches of the ulnar nerve in $100 \%$, by the medial antebrachial cutaneous nerve in $91 \%$, by volar branches directly from the ulnar nerve in $73 \%$, by the anterior interosseous nerve in $27 \%$, by the posterior interosseous nerve in $18 \%$, and by volar cutaneous branches of the median nerve in $9 \%$ [7, 16, 24].

The microscopic innervation of ligaments is characterized by specific sensory nerve endings, which can be classified regarding typical morphologic features and neurophysiologic traits according to Freeman and Wyke [4]. Ruffini endings, type I, are responsible for joint position sense. Pacini corpuscles, type II, react to joint acceleration or deceleration. Golgi-like endings, type III, respond to extreme ROM. Free nerve endings, type IV, function as nociceptors. Corpuscles, which cannot be classified as Ruffini, Pacini, Golgi-like endings, or free nerve endings, are regarded as unclassifiable corpuscles, type $\mathrm{V}$ [8].

A previous histologic analysis of the triangular fibrocartilage, separated in five areas according to dorsal, palmar, ulnar, radial, or central position, showed that the central area is noninnervated, but the periphery of the triangular fibrocartilage has all types of sensory nerve endings [2]. However, a gold chloride technique was used, which impregnates not only nerve tissue, but also elastic fibers in blood vessels and reticular fibers, thus providing nonspecific imaging of neural elements in tissue $[6,15,25]$. Furthermore, the seven different structures of the triangular fibrocartilage complex were not separately dissected. A thorough analysis of the distribution of sensory nerve endings in the triangular fibrocartilage complex may provide more insight into the proprioceptive function of these structures and, consequently, their role in the neuromuscular stability of the distal radioulnar joint, which may allow conclusions for their treatment for injured, degenerative, and painful clinical conditions.

Therefore, the aim of our study was to investigate the pattern and type of sensory nerve endings and the overall vascularity in the seven structures of the triangular fibrocartilage complex using designated immunohistochemical markers. Specifically, we aimed to (1) analyze the general 
distribution of sensory nerve endings and blood vessels; (2) examine interstructural distribution of sensory nerve endings and blood vessels; (3) compare the numbers and types of mechanoreceptors in each part; (4) analyze intrastructural distribution of nerve endings at different tissue depth; and (5) correlate our findings of triangular fibrocartilage complex neuroanatomy to known clinical entities.

\section{Materials and Methods}

\section{Cadaver Specimens}

All protocols in this study were approved by the local ethics committee review board. Eleven wrists from nine subjects, six left and five right hands, with a mean age of 80 \pm 9.8 years (range, 68-100 years) were included in this study. The wrists were refrigerated $\left(4^{\circ} \mathrm{C}\right)$ pending harvest of the triangular fibrocartilage complex. All were assessed macroscopically and radiographically to exclude posttraumatic changes, arthritis, and/or bony lesions. The triangular fibrocartilage complex with its seven parts, namely the subsheath of the extensor carpi ulnaris, the ulnocarpal meniscoid, the articular disc, and the dorsal and volar radioulnar, ulnolunate, and ulnotriquetral ligaments, were harvested (Fig. 1). The dorsal and volar radioulnar ligaments were harvested with inclusion of their bony insertions at the sigmoid notch and the ulnar styloid.

Immunohistochemistry

Specimens were immediately fixed in $4 \%$ buffered formaldehyde solution ( $\mathrm{pH}=7.4$ ), decalcified with EDTA, and embedded in paraffin. Sections of $4 \mu \mathrm{m}$ were cut and mounted on silane-coated slides for conventional staining and immunohistochemistry. All specimens were cut at five levels with a $50-\mu \mathrm{m}$ cutting interval between each level. Anti-S100 (S100; working dilution: 1:500; code Z 0311; DakoCytomation, Glostrup, Denmark), antinerve growth factor receptor p75 (working dilution: 1:200; code N-3908; Sigma, St Louis, MO, USA), and anti-Protein Gene Product 9.5 (PGP 9.5) (working dilution: 1:500; code: 78630504; AbD Serotec, Düsseldorf, Germany) were used to identify sensory nerve endings. Blood vessels were investigated using antihuman smooth muscle-actin (working dilution: 1:750; Code: M 0851; DakoCytomation). Details regarding the immunohistochemical protocol, a description of antibodies, and control stainings performed were discussed in a previous study [21].

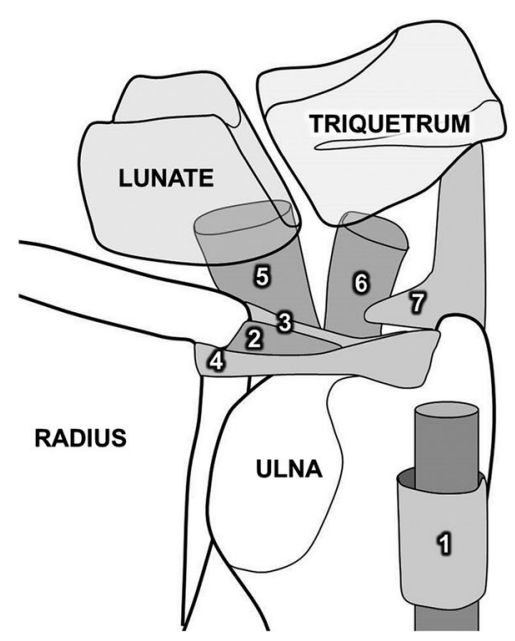

A

Fig. 1A-E (A) The triangular fibrocartilage complex consists of seven separate structures, namely the subsheath of the extensor carpi ulnaris tendon sheath $(1=\mathrm{SS}-\mathrm{ECU})$, the articular disc $(2=\mathrm{AD})$, the volar radioulnar ligament $(3=$ VRUL $)$, the dorsal radioulnar ligament (4 = DRUL), the ulnolunate ligament $(5=\mathrm{UL})$, the ulnotriquetral ligament $(6=\mathrm{UTq})$, and the ulnocarpal meniscoid $(7=\mathrm{UCM})$. $(\mathbf{B})$ The subsheath of the extensor ulnaris tendon sheath is seen after removal of the extensor retinaculum. (C) Parts 2 through 6 of the triangular fibrocartilage complex are seen after removal of the carpal

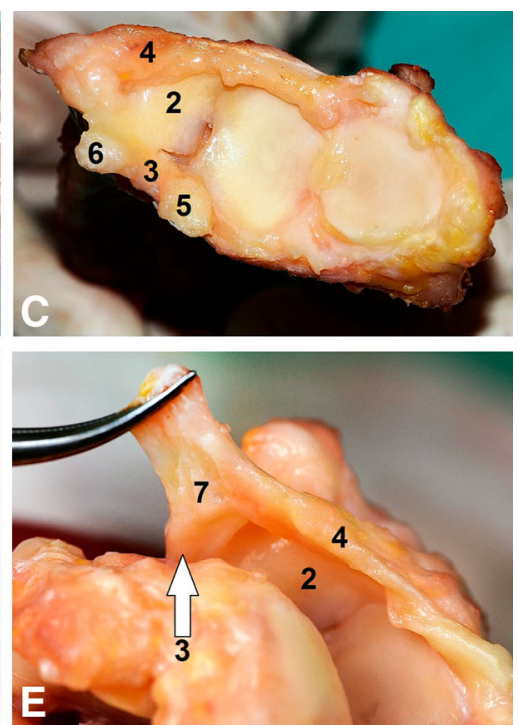

bones from the sagittal plane. (D) The ulnotriquetral ligament (6) inserts at the volar aspect of the triquetrum and originates on the radiovolar base of the ulna styloid. The ulnolunate ligament (5) inserts at the volar aspect of the lunate and originates mainly on the volar border of the articular disc (2). The dorsal radioulnar (4) and volar radioulnar (arrow 3) ligaments begin at the dorsoulnar or ulnovolar margin of the radius at the sigmoid notch and run toward the ulnar styloid. (E) A close-up view shows the ulnocarpal meniscoid (7). 
Fig. 2A-D Immunohistochemical staining of the sensory nerve endings using low-affinity nerve growth factor receptor p75 are shown. (A) The Ruffini ending is characterized by $\mathrm{p} 75$ immunoreactive dendritic nerve endings (arrowhead), a clearly visible, but non-immunoreactive central axon (white arrow), and a thin, at times partial encapsulation of the corpuscle (black arrow). (B) In contrast, the Pacini corpuscle has an onion-layered p75 immunoreactive capsule (black arrow) and central axon (white arrow). (C) The Golgi-like ending is larger with an afferent nerve fascicle (white arrow) coursing to the center of the corpuscle. Typically smaller corpuscles in the Golgilike ending are seen (arrowheads). (D) Free nerve endings (arrowhead) are p75 immunoreactive (Stain, low-affinity nerve growth factor receptor p75; original magnification, $\times 400$ ).
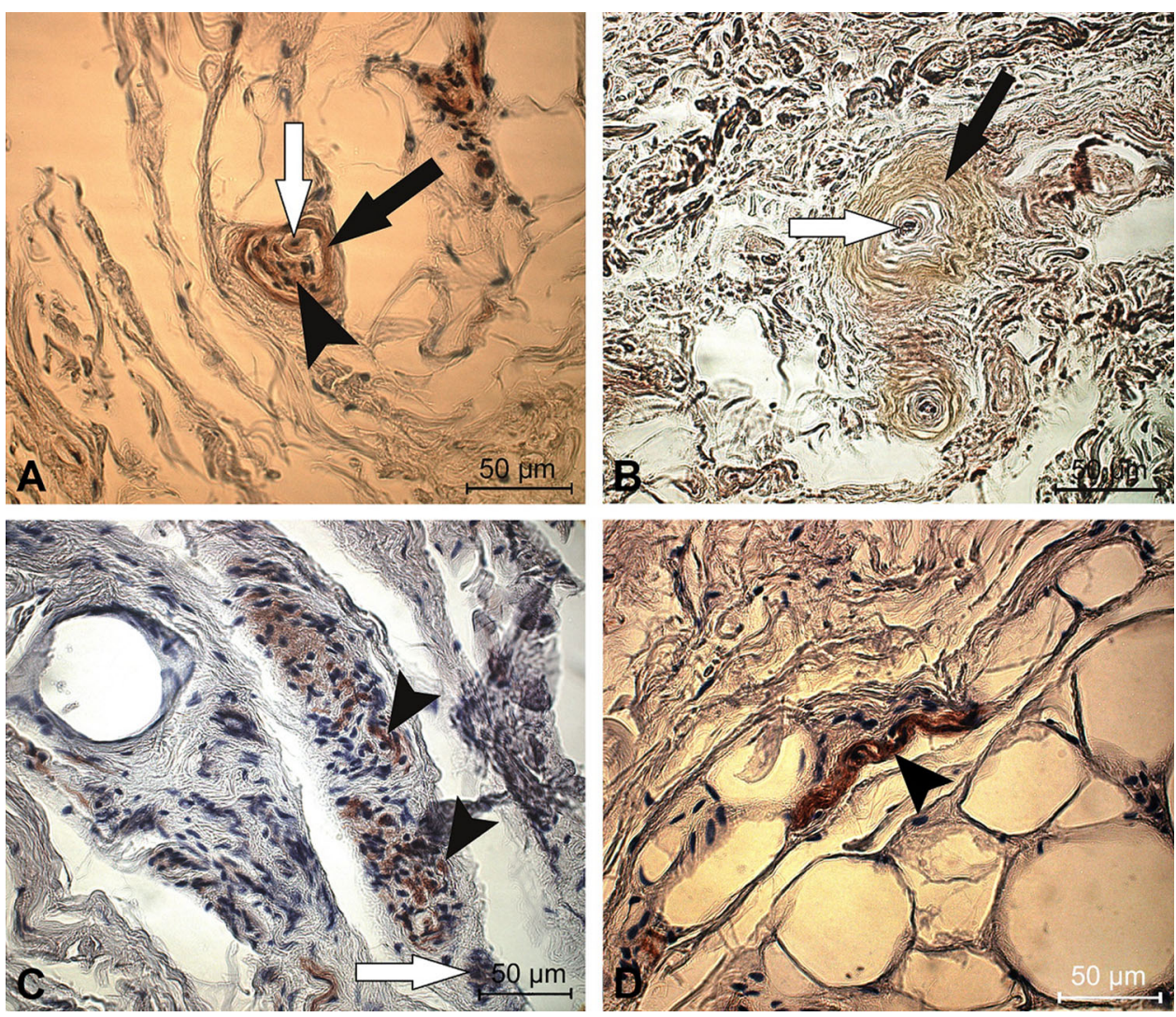

Morphologic Analysis and Cell Counting

Histologic examination of the stained tissue sections was performed using a Leica ${ }^{\circledR}$ light microscope (Leitz DMRB, Leica ${ }^{\circledR}$ Biosystems Nussloch, Germany) with a Leica ${ }^{\circledR}$ ${\text { camera }\left(\text { Leica }^{\circledR} \text { DC 300; Leica }\right.}^{\circledR}$ Microsystems CMS $\mathrm{GmbH}$, Wetzlar, Germany).

Hematoxylin and eosin-stained slices were used for determination of morphologic features of the tissue before starting the analysis of sensory nerve endings. The latter were analyzed according to the classification of Freeman and Wyke [4], modified by Hagert [8] (Fig. 2). Ruffini, Pacini, Golgi-like, free nerve endings, and unclassifiable corpuscles were counted in the S100, p75, and PGP 9.5 stainings in all five levels with respect to total cell count per section at an original magnification of $\times 400$ (highpower field). A standard $10 \times 10$ grid was used for determination of mechanoreceptor size. Sensory corpuscles, which could not be defined as Ruffini, Pacini, Golgi-like corpuscles, nor free nerve endings, were deemed as unclassifiable according to Hagert [8].

Blood vessels were counted at two representative levels in the smooth muscle-actin stain, identified by specific immunoreactivity of smooth muscle-actin of the smooth muscle cells in the wall of the vessels. All specimens were blinded for cell counts. Visual fields were counted at an original magnification of $\times 100$ to calculate the size of the analyzed slice, which is reported in $\mathrm{cm}^{2}$.

\section{Statistical Analysis}

Medians with minimum and maximum have been used for descriptive statistics throughout the article. The values represent the corpuscles $/ \mathrm{cm}^{2}$.

To analyze the general distribution of sensory nerve endings in the seven parts of the triangular fibrocartilage complex, the Friedman test was performed followed by the Wilcoxon with post hoc Bonferroni adjustments. The level of significance was set at a $p$ of 0.005 or less owing to five different types of mechanoreceptors resulting in 10 test comparisons.

To examine the interstructural distribution of sensory nerve endings among the seven parts of the triangular fibrocartilage complex, a Kruskal-Wallis test was performed followed by Mann-Whitney test with post hoc Bonferroni adjustments. The Kolmogorov-Smirnov test found that the data did not have a normal distribution. The final significance level was set at a $p$ of 0.0024 or less because of 
Table 1. Multiple comparisons

\begin{tabular}{|c|c|c|c|c|}
\hline Receptor type & Ruffini & Golgi & Pacini & Unclassifiable \\
\hline Free nerve endings & $\begin{array}{l}\text { Difference of medians } \\
72.6, p<0.001\end{array}$ & $\begin{array}{l}\text { Difference of medians, } \\
\quad p=72.6 ; p<0.001\end{array}$ & $\begin{array}{l}\text { Difference of medians, } \\
\quad p=72.6 ; p<0.001\end{array}$ & $\begin{array}{l}\text { Difference of medians, } \\
\quad p=72.6 ; p<0.001\end{array}$ \\
\hline Ruffini & & $\begin{array}{l}\text { Difference of medians } 0 \\
\quad p=0.81\end{array}$ & $\begin{array}{l}\text { Difference of medians } 0 \\
p=0.775\end{array}$ & $\begin{array}{l}\text { Difference of medians } 0, \\
\quad p=0.01\end{array}$ \\
\hline Golgi & & & $\begin{array}{l}\text { Difference of medians } 0 \\
p=0.59\end{array}$ & $\begin{array}{l}\text { Difference of medians } 0, \\
p=0.015\end{array}$ \\
\hline Pacini & & & & $\begin{array}{l}\text { Difference of medians } 0, \\
p=0.011\end{array}$ \\
\hline
\end{tabular}

Table 2. Relationship between the sensory nerve endings*

\begin{tabular}{ll}
\hline Sensory nerve endings & Relationship \\
\hline Ruffini: Pacini & $1.33: 1$ \\
Ruffini: Golgi & $1.29: 1$ \\
Ruffini: unclassifiable & $1: 1.72$ \\
Pacini: Golgi & $1: 1.03$ \\
Pacini: unclassifiable & $1: 2.3$ \\
Golgi: unclassifiable & $1: 2.2$ \\
Free nerve endings: Ruffini & $497: 1$ \\
Free nerve endings: Pacini & $661: 1$ \\
Free nerve endings: Golgi & $644: 1$ \\
Free nerve endings: unclassifiable & $287: 1$ \\
Free nerve endings: blood vessels & $1: 2.4$
\end{tabular}

* Calculated with the mean of all parts of the triangular fibrocartilage complex $(\mathrm{n}=77)$.

21 tests among the seven parts of the triangular fibrocartilage complex.

To compare the total number and types of mechanoreceptors in each part of the triangular fibrocartilage complex, Fisher's exact test was performed with significance set at $\mathrm{p}$ of 0.05 or less.

To analyze the intrastructural distribution of sensory nerve endings in each part of the triangular fibrocartilage complex among the five levels studied, to assess whether the sensory nerve endings are distributed homogenously, a Kruskal-Wallis test followed by the Mann-Whitney test with post hoc Bonferroni adjustments was used. The five levels allowed 10 possible test comparisons, which results in a significance level of $\mathrm{p}$ of 0.005 or less.

\section{Results}

General Distribution

Free nerve endings (median, 72.6; range, 0-469.4/ $\mathrm{cm}^{2}$ ) were the predominant mechanoreceptor (Table 1) and were more common than all other types of sensory nerve endings ( $\mathrm{p}<0.001$, respectively) followed by unclassifiable

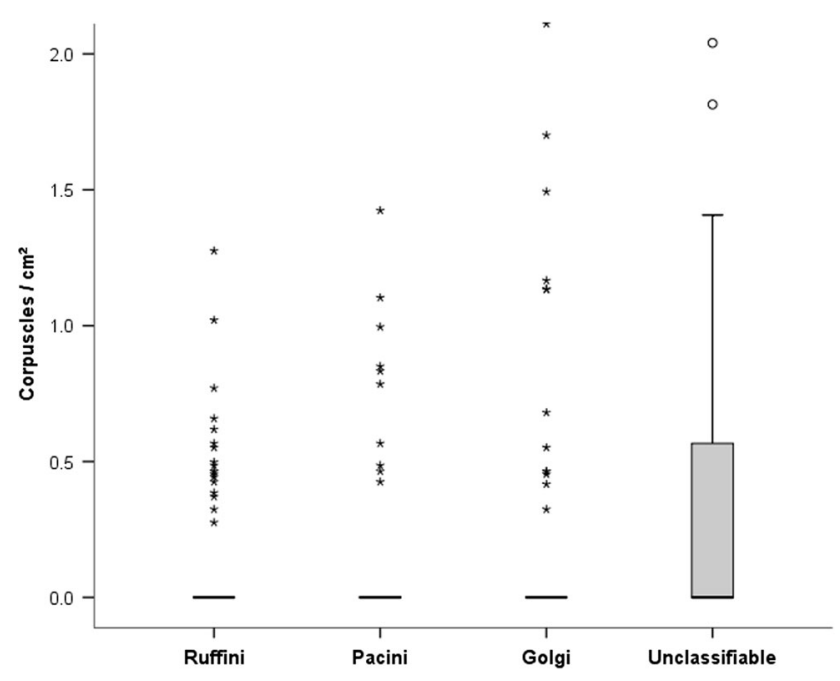

Fig. 3 The general distributions of sensory nerve endings are shown as medians with minimum and maximum. There were no significant differences between all sensory corpuscles. $*=$ values, which are more than 1.5-fold away from minimum or maximum; $o=$ values, which are more than threefold away from minimum or maximum.

corpuscles (median, 0; range, $0-2.5 / \mathrm{cm}^{2}$ ), Ruffini endings (median, 0; range, $0-5.6 / \mathrm{cm}^{2}$ ), Golgi-like endings (median, 0; range, 0-2.1 / $\mathrm{cm}^{2}$ ), and Pacini corpuscles (median, 0; range, $0-3.8 / \mathrm{cm}^{2}$ ) (Table 2; Fig. 3). More blood vessels (median, 186.6; range, $0-1090.6 / \mathrm{cm}^{2}$ ) than free nerve endings (median, 72.6; range, 0-469.4/ $/ \mathrm{cm}^{2}$; difference of medians 114; $\mathrm{p}<0.001$ ) were observed (Fig. 4).

\section{Interstructural Distribution and Mechanoreceptor Types}

All types of sensory corpuscles were found in the subsheath of the extensor carpi ulnaris, the ulnocarpal meniscoid, dorsal and volar radioulnar ligaments, and ulnotriquetral ligaments. However, the ulnolunate ligament did not contain Ruffini nor Pacini corpuscles, and the articular disc lacked all corpuscles except for free nerve endings, which was present only in its ulnar part. As for unclassifiable corpuscles, the articular disc $\left(0\right.$; range, $\left.0-0 / \mathrm{cm}^{2}\right)$ had fewer numbers than the subsheath of the extensor carpi ulnaris 


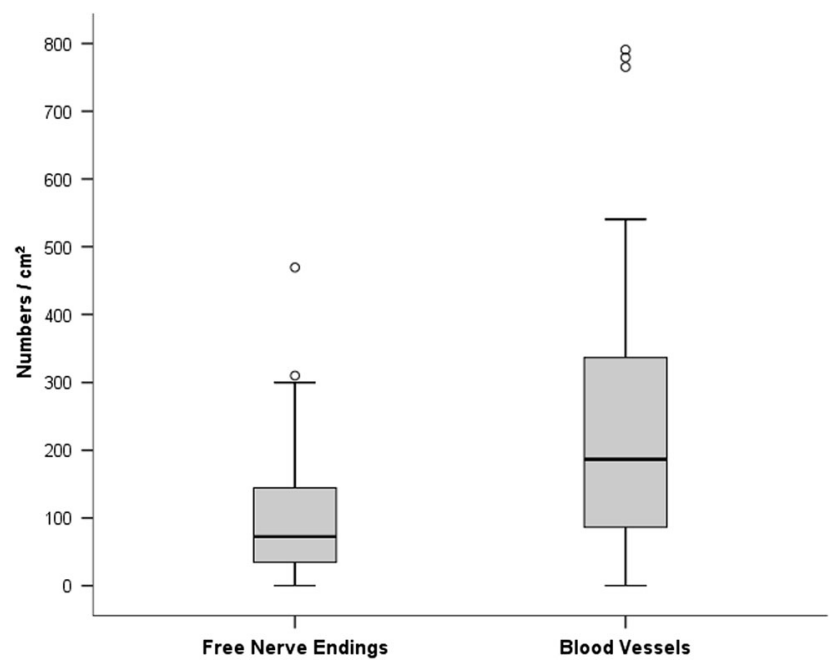

Fig. 4 The general distributions of free nerve endings and blood vessels are shown as medians with minimum and maximum. There were significantly more blood vessels than free nerve endings $(\mathrm{p}<$ 0.001). $\mathrm{o}=$ values, which are more than threefold away from minimum or maximum.

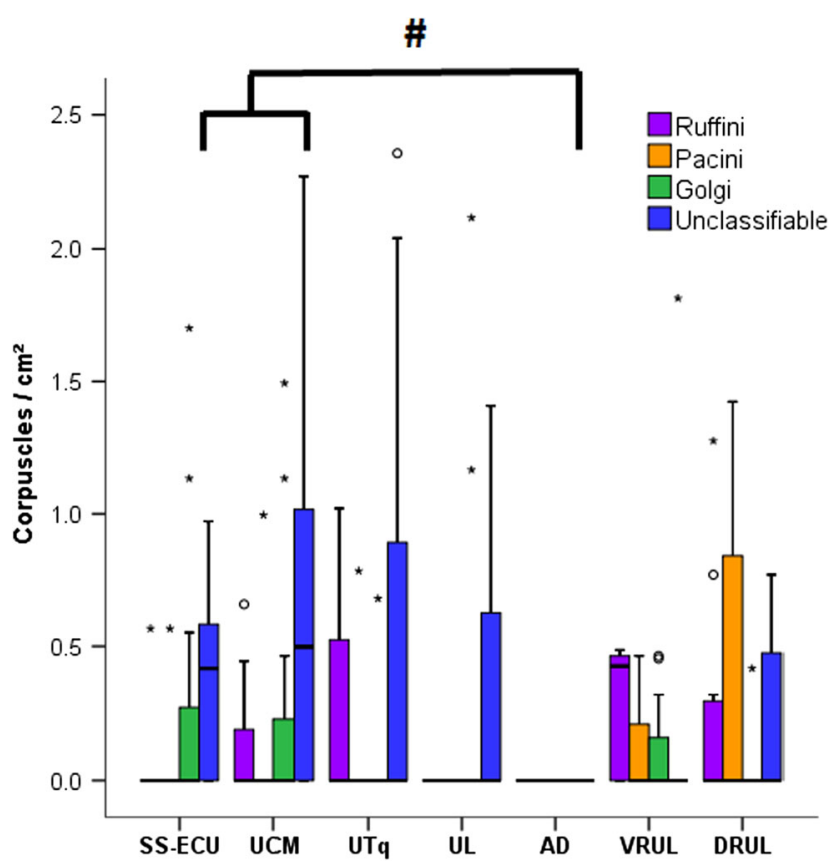

Fig. 5 Medians with minimum and maximum for the sensory nerve endings are shown for each part of the triangular fibrocartilage complex. The subsheath of the extensor carpi ulnaris (SS-ECU) and the ulnocarpal meniscoid (UCM) had significantly more unclassifiable corpuscles than the articular disc (AD) $\left({ }^{\#} \mathrm{p}=0.002\right)$. All other comparisons of the sensory nerve endings between the seven different structures of the triangular fibrocartilage complex were not statistically significant. UTq = ulnotriquetral ligament; UL = ulnolunate ligament; VRUL $=$ volar radioulnar ligament; DRUL $=$ dorsal radioulnar ligament; $*$ = values, which are more than 1.5 -fold away from minimum or maximum; $\mathrm{o}=$ values, which are more than threefold away from minimum or maximum. (median, 0.42; range, $0-0.97 / \mathrm{cm}^{2}$; difference of medians $0.42 ; \mathrm{p}=0.002$ ) and the ulnocarpal meniscoid (median, 0.49 ; range, $0-2.55 / \mathrm{cm}^{2}$; difference of medians, $0.49 ; \mathrm{p}=$ 0.002) (Fig. 5). As for free nerve endings and blood vessels, the articular disc contained fewer free nerve endings (median, 7.74; range, 0-72.65 / $/ \mathrm{cm}^{2}$ ) and fewer blood vessels (median, 29.86; range, $0-112.24 / \mathrm{cm}^{2}$ ) than all other structures of the triangular fibrocartilage complex ( $\mathrm{p} \leq$ 0.001) except the ulnolunate ligament (Fig. 6).

Regarding blood vessels of the volar radioulnar ligament (median, 363.62; range, $117.8-871.17 / \mathrm{cm}^{2}$ ) had a higher number than the ulnolunate ligament (median, 107.71; range, 15.94-410.33/ $\mathrm{cm}^{2}$; difference of medians, 255.91; $\mathrm{p}$ $=0.002$ ) and the dorsal radioulnar ligament (median, 116.15; range, $53.91-185.1 / \mathrm{cm}^{2}$; difference of medians, 247.47; $\mathrm{p}=0.001$ ) (Fig. 6).

\section{Comparison of Mechanoreceptor Type in Each} Structure

Free nerve endings were obtained in each structure more often than all other types of sensory nerve endings. The medians and ranges are: for the subsheath of the extensor carpi ulnaris (free nerve endings: median, 66.3, range, 34.9$267 / \mathrm{cm}^{2}$; Ruffini: median, 0 , range, $0-0.6 / \mathrm{cm}^{2}$, difference of medians, 66.3, $\mathrm{p}<0.001$; Pacini: median, 0, range, $0-0.6 /$ $\mathrm{cm}^{2}$, difference of medians, 66.3, $\mathrm{p}<0.001$; Golgi-like: median, 0 , range, $0-1.7 / \mathrm{cm}^{2}$, difference of medians, $66.3, \mathrm{p}<$ 0.001 ; and unclassifiable: median, 0.4 , range, $0-1 / \mathrm{cm}^{2}$, difference of medians, 65.9, $\mathrm{p}<0.001$ ); for the ulnocarpal meniscoid (free nerve endings: median, 126.9, range, 34.8$298.2 / \mathrm{cm}^{2}$; Ruffini: median, 0 , range, $0-0.7 / \mathrm{cm}^{2}$, difference of medians, 126.9; Pacini: median, 0, range, $0-1 / \mathrm{cm}^{2}$, difference of medians, 126.9; Golgi-like: median, 0 , range, 0 $1.5 / \mathrm{cm}^{2}$, difference of medians, 126.9 ; and unclassifiable: median, 0.5 , range, $0-2.6 / \mathrm{cm}^{2}$, difference of medians, 126.4); for the articular disc (free nerve endings: median, 7.7, range, $0-72.6 / \mathrm{cm}^{2}$; Ruffini: median, 0 , range, $0-0 / \mathrm{cm}^{2}$, difference of medians, 7.7; Pacini: median, 0 , range, $0-0 / \mathrm{cm}^{2}$, difference of medians, 7.7; Golgi-like: median, 0, range, 0-0/ $\mathrm{cm}^{2}$, difference of medians, 7.7; and unclassifiable: median, 0 , range, $0-0 / \mathrm{cm}^{2}$, difference of medians, 7.7); for the distal radioulnar ligament (free nerve endings: median, 82.3, range, 19.6-151.4/ $\mathrm{cm}^{2}$; Ruffini: median, 0, range, 0-1.3/ $\mathrm{cm}^{2}$, difference of median, 82.3; Pacini: median, 0, range, 0 $1.4 / \mathrm{cm}^{2}$, difference of medians, 82.3 ; Golgi-like: median, 0 , range, $0-0.4 / \mathrm{cm}^{2}$, difference of medians, 82.3 ; and unclassifiable: median, 0 , range, $0-0.8 / \mathrm{cm}^{2}$, difference of medians, 82.3); for the volar radioulnar ligament (free nerve endings: median, 170.3 , range, $23.4-310.2 / \mathrm{cm}^{2}$; Ruffini: median, 0.4 , range, $0-5.6 / \mathrm{cm}^{2}$, difference of medians, 169.9; Pacini: median, 0 , range, $0-3.8 / \mathrm{cm}^{2}$, difference of medians, 170.3 ; 
Fig. 6 The distributions of free nerve endings and blood vessels are seen as medians with minimum and maximum. The subsheath of the extensor carpi ulnaris (SS-ECU), ulnocarpal meniscoid (UCM), ulnotriquetral ligament (UTq), and volar (VRUL) and dorsal radioulnar ligaments (DRUL) had significantly more free nerve endings and blood vessels than the articular disc (AD) $(* \mathrm{p}<0.001$, respectively). Furthermore, the volar radioulnar ligament contained significantly more blood vessels than the ulnolunate $\left({ }^{\S} \mathrm{p}=\right.$ 0.002 ) and dorsal radioulnar ligaments $\left({ }^{*} \mathrm{p}=0.001\right)$. $*$ = values, which are more than 1.5 -fold away from minimum or maximum; $0=$ values, which are more than threefold away from minimum or maximum.

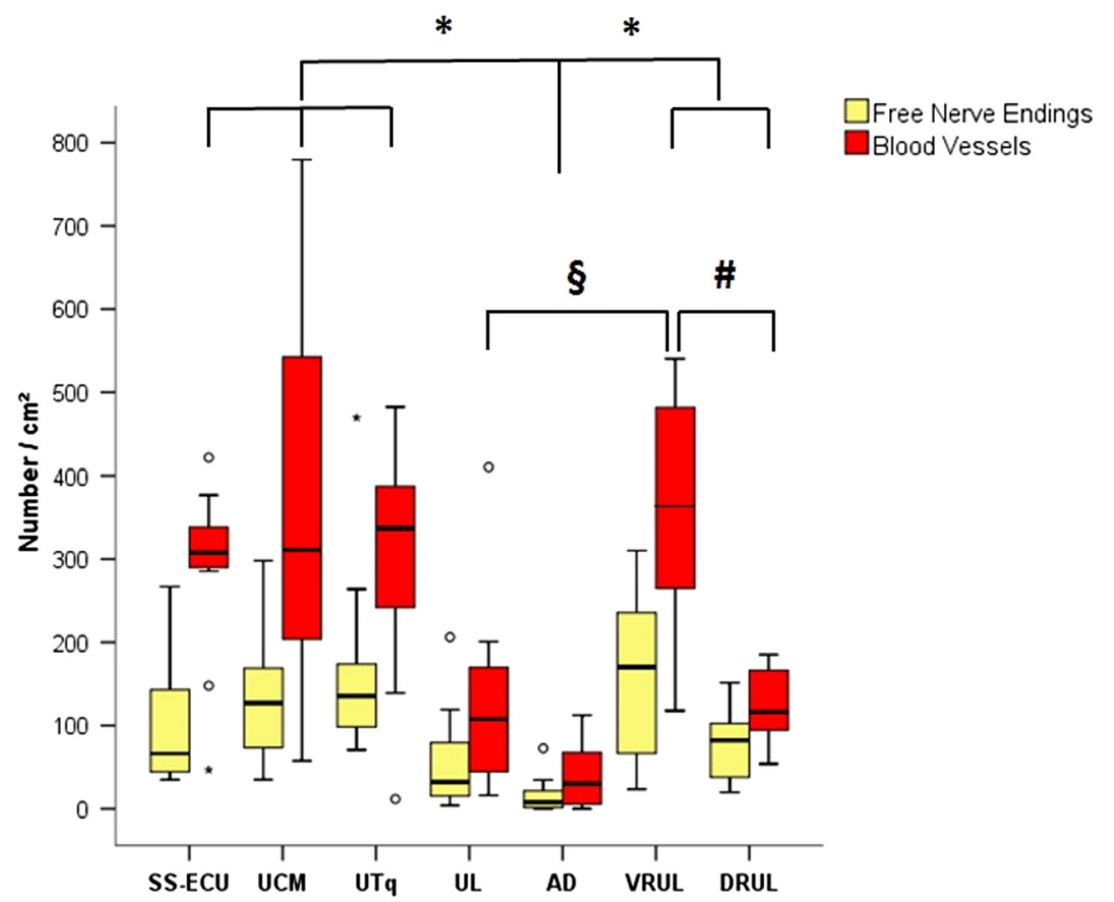

Golgi-like: median, 0 , range, $0-0.5 / \mathrm{cm}^{2}$, difference of medians, 170.3; and unclassifiable: median, 0 , range, $0-1.8 /$ $\mathrm{cm}^{2}$, difference of medians, 170.3); for the ulnolunate ligament (free nerve endings: median, 31.9, range, 4.1-206.3/ $\mathrm{cm}^{2}$; Ruffini: median, 0 , range, $0-0 / \mathrm{cm}^{2}$, difference of medians, 31.9; Pacini: median, 0 , range, $0-0 / \mathrm{cm}^{2}$, difference of medians, 31.9; Golgi-like: median, 0, range, $0-2.1 / \mathrm{cm}^{2}$, difference of medians, 31.9; and unclassifiable: median, 0 , range, $0-1.4 / \mathrm{cm}^{2}$, difference of medians, 31.9 ); and for the ulnotriquetral ligament (free nerve endings: median, 135.4, range, 70.6-469.4/ $\mathrm{cm}^{2}$; Ruffini: median, 0 , range, $0-1 / \mathrm{cm}^{2}$, difference of medians, 135.4; Pacini: median, 0, range, 0 $0.8 / \mathrm{cm}^{2}$, difference of medians, 135.4; Golgi-like: median, 0 , range, $0-0.7 / \mathrm{cm}^{2}$, difference of medians, 135.4 ; and unclassifiable: median, 0 , range, $0-2.4 / \mathrm{cm}^{2}$, difference of medians, 135.4).

\section{Intrastructural Distribution at Different Tissue Depths}

The intrastructural analysis revealed no differences in mechanoreceptor distribution in all investigated specimens with the numbers available, showing a homogenous distribution of proprioceptive qualities in all seven parts of the triangular fibrocartilage complex.

\section{Discussion}

\section{Background, Rationale, Aims}

The triangular fibrocartilage complex plays a fundamental role in stabilization of the distal radioulnar joint. The dorsal and volar radioulnar ligaments are the most studied components of the triangular fibrocartilage complex as they are the principle stabilizers of the distal radioulnar joint $[5,27]$. However, the triangular fibrocartilage complex consists of seven different structures and their sensory role in dynamic distal radioulnar joint stabilization is unclear. Sensory nerve endings react on mechanical stimuli, for example, joint position change, transforms them in neural excitations, which are sent to the spinal cord where a part of this information is sent directly to the muscles which stabilize the joint. Another part of the information is sent to the central nervous system for central integration and processing. The different types of sensory nerve endings are assigned different functions as outlined in the introduction. In the current study, the sensory nerve endings of the seven parts of the triangular fibrocartilage complex were examined in detail.

\section{Limitations}

Unclassifiable corpuscles were the second most common mechanoreceptor type, suggesting a high morphologic variety of sensory nerve endings, also observed in other studies $[18,26]$. It also points to a possible limitation of the existing gold standard classification of Freeman and Wyke [4], modified by Hagert [8], because many sensory nerve endings apparently will not fit into the established and limited classification schemes. The high number of unclassifiable corpuscles is also a limitation of the applied descriptive analysis using two-dimensional light- 
microscopy, which does not permit a complete representation of the whole mechanoreceptor [21]. Nevertheless, even three-dimensional reconstructions of multiple levels of ligaments have revealed a large portion of unclassifiable corpuscles in wrist ligaments [26].

\section{General Distribution}

Free nerve endings were the predominant mechanoreceptor type in all of the examined structures of the triangular fibrocartilage complex, which is in accordance with a previous study [24] and studies of the human ankle [21], fat tissue in the human sinus tarsi [23], and the human knee capsule [12]. The predominance of free nerve endings indicates an important role in perceiving noxious, chemical, mechanical, and inflammatory stimuli. Ruffini endings were the third most common mechanoreceptors closely followed by Golgi-like endings and Pacini corpuscles. A study on ligaments at the ankle revealed higher amounts of Ruffini endings followed by Pacini corpuscles and Golgilike endings with the following ratios: Ruffini: Pacini = 4.7:1; Ruffini: Golgi $=15.5: 1$, and Pacini: Golgi $=3.3: 1$ [21]. The more equal relationships among Ruffini, Pacini, and Golgi-like endings in the current study (Table 2) indicate that the ulnocarpal and distal radioulnar joints require a homogenous distribution of proprioceptive qualities.

\section{Interstructural Distribution and Mechanoreceptor Types}

The subsheath of the extensor carpi ulnaris, the ulnocarpal meniscoid, the volar radioulnar ligament, the dorsal radioulnar ligament, and the ulnotriquetral ligament were all richly innervated, containing all types of sensory nerve endings. In contrast, the ulnolunate ligament was sparsely innervated with only Golgi-like endings, free nerve endings, and unclassifiable corpuscles. The articular disc had only free nerve endings in its ulnar portion. This is in contrast to Calvacante et al. [2], who reported that the central area was noninnervated, but the periphery of the articular disc had all types of sensory nerve endings. The primary difference between our study and that of Calvacante et al. [2] is that we mapped the mechanoreceptor population using a combination of immunohistochemical markers: S-100 protein, the neurotrophic receptor $\mathrm{p} 75$, and PGP 9.5. These three antibodies allow precise differentiation of mechanoreceptors owing to selective delineation of specific neural and perineural structures in sensory nerve endings [22]. However, gold chloride impregnates not only nerve tissue, but also elastic fibers in blood vessels and reticular fibers, thus providing nonspecific imaging of neural elements in tissue [6]. Furthermore, in the current study, all seven parts of the triangular fibrocartilage complex were dissected separately and analyzed in five levels, respectively. An additional study of innervation of the triangular fibrocartilage complex only detected free nerve endings, but no Ruffini, Pacini, or Golgi-like corpuscles, using PGP 9.5 and calcitonin gene-related peptide [24]. However, the analyzed sections had a thickness of $100 \mu \mathrm{m}$, and the amount of each neural element was counted in randomly selected areas of each structure at only one level.

\section{Intrastructural Analysis}

No differences have been observed among the five levels of each structure, which indicates a homogenous distribution of sensory nerve endings resulting in a stable and consistent innervation pattern. A recent study investigating ligaments around the ankle in five levels had similar findings [22]. Therefore, a partial ligament rupture may not result in complete ligamentous denervation.

\section{Clinical Relevance}

The greatest amount of Ruffini endings was observed in the volar radioulnar ligament, indicating that this ligament has an important role in detecting joint position during forearm rotation. The radioulnar ligaments are the primary static stabilizers of the distal radioulnar joint and, as we found, also are important for neuromuscular stability with their consistently rich innervation. Patients with distal radioulnar joint instabilities attributable to foveal tears should be treated primarily with open or arthroscopic reattachments to restore static and dynamic distal radioulnar joint stability [17]. The inclusion of proprioceptive exercises after anatomic reattachment of the volar and dorsal radioulnar ligaments is recommended during the postoperative followup, after the required period of immobilization for ligament healing has passed [9]. Contrarily, surgical procedures aiming to reconstruct the radioulnar ligaments with a tendon graft [1], will not address the sensory function of the volar and dorsal radioulnar ligaments, and consequently fail to account for the dynamic aspects of distal radioulnar joint stability [13].

Free nerve endings were the predominant mechanoreceptor type followed by unclassifiable corpuscles and then by a fairly equal distribution of Ruffini, Pacini, and Golgilike corpuscles. Nociception therefore must be considered as having great importance in the proprioceptive and neuromuscular control of the distal radioulnar joint. The articular disc and ulnolunate ligament were rarely innervated, indicating a primarily mechanical function, whereas 
the subsheath of the extensor carpi ulnaris, ulnocarpal meniscoid, volar and dorsal radioulnar ligaments, and ulnotriquetral ligament, with their rich innervations, have distinct proprioceptive qualities. Lesions of the volar and dorsal radioulnar ligaments have immense consequences not only for mechanical but also for dynamic stability of the distal radioulnar joint, and surgical reconstruction of a radioulnar ligament injury is of great importance.

Acknowledgments We thank Ursula Range (Institute for Medical Informatics and Biometry, University Hospital "Carl Gustav Carus", Dresden, Germany) for statistical support, Suzanne Manthey and Dorothea Liebeheim (both from the Center for Orthopedics and Trauma Surgery, University Hospital "Carl Gustav Carus) for histologic preparation, Thomas Albrecht (Center for Orthopedics and Trauma Surgery, University Hospital "Carl Gustav Carus) for logistic support, and Manuel Llusá Pérez MD, PhD (Department of Anatomy, University of Barcelona, Barcelona, Spain) for generous assistance in the laboratory work.

\section{References}

1. Adams BD, Berger RA. An anatomic reconstruction of the distal radioulnar ligaments for posttraumatic distal radioulnar joint instability. J Hand Surg Am. 2002;27:243-251.

2. Cavalcante ML, Rodrigues CJ, Mattar R Jr. Mechanoreceptors and nerve endings of the triangular fibrocartilage in the human wrist. J Hand Surg Am. 2004;29:432-435; discussion 436-438.

3. Frank CB. Ligament structure, physiology and function. $J$ Musculoskelet Neuronal Interact. 2004;4:199-201.

4. Freeman MA, Wyke B. The innervation of the ankle joint: an anatomical and histological study in the cat. Acta Anat (Basel). 1967;68:321-333.

5. Garcia-Elias M. Soft-tissue anatomy and relationships about the distal ulna. Hand Clin. 1998;14:165-176.

6. Gomez-Barrena E, Martinez-Moreno E, Ballesteros Masso R, Martinez Perez D, Munuera Martinez L. Gold chloride technique to study articular innervation: a protocol validated through computerassisted colorimetry. Histol Histopathol. 1999;14:69-79.

7. Gupta R, Nelson SD, Baker J, Jones NF, Meals RA. The innervation of the triangular fibrocartilage complex: nitric acid maceration rediscovered. Plast Reconstr Surg. 2001;107:135-139.

8. Hagert E. Wrist ligaments: innervation patterns and ligamentomuscular reflexes. PhD thesis. Stockholm, Sweden: Karolinska Institutet; 2008. Available at: https://publications.ki.se/xmlui/ bitstream/handle/10616/39802/thesis.pdf?sequence $=1$. Accessed May 4, 2015.

9. Hagert E. Proprioception of the wrist joint: a review of current concepts and possible implications on the rehabilitation of the wrist. J Hand Ther. 2010;23:2-16; quiz 17.

10. Hagert E, Chim H, Moran SL. Anatomy of the distal radioulnar joint and ulnocarpal complex. In Greensberg JA, ed. Ulnar-Sided
Wrist Pain: A Master Skills Publication. Chicago, IL: American Society for Surgery of the Hand (ASSH) e-Books; 2013:11-21.

11. Hagert E, Hagert CG. Understanding stability of the distal radioulnar joint through an understanding of its anatomy. Hand Clin. 2010;26:459-466.

12. Halata Z, Rettig T, Schulze W. The ultrastructure of sensory nerve endings in the human knee joint capsule. Anat Embryol (Berl). 1985;172:265-275.

13. Kim SH, Chun $\mathrm{CH}$, Chun KC, Jo HJ, Kim KM. Histological assessment of mechanoreceptors in Achilles allografts after anterior cruciate ligament reconstruction. Am J Sports Med. 2012;40:2061-2065.

14. Kleinman WB. Stability of the distal radioulnar joint: biomechanics, pathophysiology, physical diagnosis, and restoration of function. What we have learned in 25 years. J Hand Surg Am. 2007;32:1086-1106.

15. Koch B, Kurriger G, Brand RA. Characterisation of the neurosensory elements of the feline cranial cruciate ligament. $J$ Anat. 1995;187 (pt 2):353-359.

16. LaPorte DM, Hashemi SS, Dellon AL. Sensory innervation of the triangular fibrocartilage complex: a cadaveric study. J Hand Surg Am. 2014;39:1122-1124.

17. Lawler E, Adams BD. Reconstruction for DRUJ instability. Hand (NY). 2007;2:123-126.

18. Mobargha N, Ludwig C, Ladd AL, Hagert E. Ultrastructure and innervation of thumb carpometacarpal ligaments in surgical patients with osteoarthritis. Clin Orthop Relat Res. 2014;472:11461154.

19. Palmer AK. Triangular fibrocartilage complex lesions: a classification. J Hand Surg Am. 1989;14:594-606.

20. Palmer AK, Werner FW. The triangular fibrocartilage complex of the wrist: anatomy and function. J Hand Surg Am. 1981;6:153162.

21. Rein S, Hagert E, Hanisch U, Lwowski S, Fieguth A, Zwipp H. Immunohistochemical analysis of sensory nerve endings in ankle ligaments: a cadaver study. Cells Tissues Organs. 2013;197:6476.

22. Rein S, Hanisch U, Zwipp H, Fieguth A, Lwowski S, Hagert E. Comparative analysis of inter- and intraligamentous distribution of sensory nerve endings in ankle ligaments: a cadaver study. Foot Ankle Int. 2013;34:1017-1024.

23. Rein S, Manthey S, Zwipp H, Witt A. Distribution of sensory nerve endings around the human sinus tarsi: a cadaver study. $J$ Anat. 2014;224:499-508.

24. Shigemitsu T, Tobe M, Mizutani K, Murakami K, Ishikawa Y, Sato F. Innervation of the triangular fibrocartilage complex of the human wrist: quantitative immunohistochemical study. Anat $S c i$ Int. 2007;82:127-132.

25. Soule JD. Direct staining of reticular fibers with gold chloride. Stain Technol. 1962;37:31-34.

26. Tomita K, Berger EJ, Berger RA, Kraisarin J, An KN. Distribution of nerve endings in the human dorsal radiocarpal ligament. J Hand Surg Am. 2007;32:466-473.

27. Weaver L, Tencer AF, Trumble TE. Tensions in the palmar ligaments of the wrist: I. The normal wrist. J Hand Surg Am. 1994;19:464-474. 